ISSN: 2576-8875

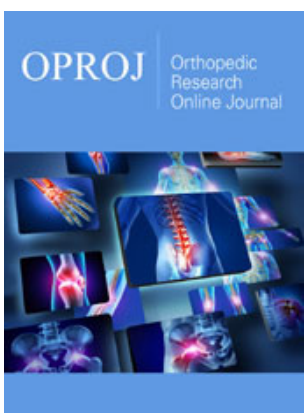

*Corresponding author: Christos Tsigkanos, Department of Medicine, National and Kapodistrian University of Athens, Greece

Submission: 監 July 17, 2020

Published: 㘹July 27, 2020

Volume 7 - Issue 3

How to cite this article: Christos Tsigkanos, Theano Demestiha, Chara Spiliopoulou, Georgios Tsigkanos. Movement Variability in Gait: Low Back Pain Kinematics and Chaos. Ortho Res Online J. 7(3). OPROJ. 000664. 2020.

DOI: 10.31031/OPROJ.2020.07.000664

Copyright@: Christos Tsigkanos, This article is distributed under the terms of the Creative Commons Attribution 4.0 International License, which permits unrestricted use and redistribution provided that the original author and source are credited.

\section{Movement Variability in Gait: Low Back Pain Kinematics and Chaos}

\author{
Christos Tsigkanos $^{1 *}$, Theano Demestiha ${ }^{1}$, Chara Spiliopoulou ${ }^{1}$ and Georgios \\ Tsigkanos $^{2}$ \\ ${ }^{1}$ Department of Medicine, National and Kapodistrian University of Athens, Greece \\ ${ }^{2}$ Department of Physical Education and Sport Science, National and Kapodistrian University \\ of Athens, Greece
}

\begin{abstract}
Introduction: Kinematic analysis has been a dominant tool for addressing the neuromuscular and proprioceptive alterations that occur in Low Back Pain (LBP) patients. Movement variability is a crucial component of this analysis. During the past years, a promising approach is the application of non-linear indices.
\end{abstract}

Objective: The aim of the study was to compare movement variability, as expressed mainly by non-linear indices, at the pelvis and lumbar between LBP patients and healthy participants during gait.

A. Participants and setting: Sixteen (16) LBP patients and thirteen (13) healthy control subjects (nonathletes) participated in the study. Participants walked on a treadmill at different walking conditions while recorded by a 6-infrared camera optoelectronic system. Kinematic variability of pelvic and lumbar movement was analyzed using linear (standard deviation) and non-linear indices (Maximal Lyapunov Exponent- LyE and Approximate Entropy- ApEn).

B. Main outcome measures: It is expected that the LBP group will present with lower ApEn and/ or LyE values, underlying the less chaotic nature of their gait pattern. Gender differences in movement variability will also be investigated.

Results: Healthy subjects were found to have significantly greater mean values than LBP patients at seven pelvic and lumbar components in LyE, ApEn and SD. Specifically the calculated LyE at the pelvis during normal gait was proven to have a sensitivity of $92.3 \%$ and a specificity of $90 \%$ in the discrimination of healthy subjects from LBP patients. Female subjects presented with higher variability than males.

Conclusion: Healthy participants presented with higher movement variability indicating a more chaotic kinematic behavior in comparison to LBP patients. Lower variability values may be partly explained by the attempt of LBP patients to avoid painful end of range of motion positions. In this perspective non- linear indices seem to relate to qualitive characteristics of movement that need to be taken into consideration during rehabilitation.

Keywords: Low back pain; Movement variability; Gait kinematics; Maximal lyapunov exponent; Approximate entropy

\section{Introduction}

Low Back Pain (LBP) has been the most common health problem in western countries in the last decades [1,2]. LBP incidence is $18.6 \%$ in general population while at ages between 30 and 50 years it is the most expensive health care problem $[3,4]$. Numerous parameters of everyday life, skeletal impairment, biomechanical changes and proprioceptive deficits have been scrutinized as possible causation for the chronicity and recurrence of the disease. Kinematic analysis has been a dominant tool for addressing the neuromuscular and proprioceptive alterations that occur in LBP patients [5,6]. Movement variability is a crucial component of this analysis and can be described as the physiological alterations of human performance [7]. From a certain perspective abnormal human movement variability includes all the unorthodox kinematic behavior while performing and can be minimized by continuous training [8]. Human movement can be described by chaotic complex systems with underlying patterns, constant feedback loops, repetition, self-similarity, fractals, and self-organization 
[9]. However, is all this variability unnecessary noise or is it the basis of efficient and unperturbed gait?

Linear mathematics rather fail to quantify and describe in a reliable manner the amount- levels of variability present since they average data and ban any unwanted 'noise' thus missing the temporal variation of movement structure $[10,11]$. On the contrary, non- linear tools can effectively describe these aspects as they can measure both randomness and periodicity of a time series. The most commonly non- linear statistical tools used in the description of human gait are Approximate Entropy (ApEn) and the Maximal Lyapunov Exponent (LyE). They both describe regularity and, subsequently, the amount of randomness in a time series. Higher ApEn values describe a more irregular and non- repetitive system usually with decreased movement control and neuromuscular coordination [12]. LyE is widely used in measuring gait stability $[13,14]$. It measures the structure of variation and is particularly sensitive to data with periodic nature $[10,15]$.

According to a relatively recent theoretical frame [7], every system is characterized by a state of Optimal Movement Variability (OMV) which is defined by the ideal amount of normal variations that a specific task includes with a specific chaotic structure. Less amount of variability entails a more rigid system, highly predictable and less adaptable to external perturbations, while higher variability describes a more unstable, noisy, unpredictable system. Elite athletes or musicians have the ability of successfully performing a task in many ways while a less capable individual would have little success even when attempting a much plainer task [7]. Various biological systems like heart beats, blood pressure and gait function according to chaotic rhythms [10]. Human gait should be analyzed under in terms of fractal dynamics and deterministic chaos: human gait is not limited by determinism but is constantly readapted to environmental demands in ways that cannot be foreseen [16]. Pathological gait models present either with excessive periodicity and predictability or extended randomness and irregularity $[17,18]$.

Stergiou et al. [10] investigated movement variability in 10 participants with unilateral Anterior Cruciate Ligament (ACL) deficiency during normal gait. A 6- camera Vicon Nexus system and a treadmill were used. Local knee stability in the sagittal plane was assessed by comparing the largest LyE values of the intact knee to the injured one. Subjects walked on a motorized treadmill at their self- selected speed, 20\% lower and 20\% higher to that. Regardless of the walking speed the injured knee had significantly higher LyE values indicating a more locally unstable knee, less able to adjust to random perturbations.

Zampeli et al. [19] attempted to verify the above results in backward walking. 15 ACL arthroscopically confirmed deficient patients were compared to 10 healthy subjects in backward walking at their self- selected speed. Hamstrings' activation plays an important role in gait since they initiate knee flexion and hip extension in the early swing of the gait cycle actively stabilizing the knee [20]. Backward walking imposes the joint to lower stress because of the concentric contraction of the quadriceps instead of the eccentric contraction of forward walking [21]. The maximum LyE of both knees was calculated. The control group had significantly higher LyE values than the experimental group in both knees. In addition, the intact knee of the experimental group had higher LyE values in comparison to the ACL deficient knee. The experimental group presented with a more rigid movement behavior indicating restricted neuromuscular coordination and limited adaptability in environmental perturbations [19]. Quadriceps inhibition and the loss of mechanoreceptors are the reason for these kinematic variations $[20,22]$. The lower LyE of the intact knee of the experimental group is attributed to biomechanical changes of the entire limb [10,23].

Steele et al. [24] investigated movement variability of the lumbar spine using a 10 camera 3- D analysis system in normal gait (8 meters distance) after applying a 12- week muscle strengthening program of lumbar extensors once a week. The outcome measures included angular displacement, kinematic waveform pattern $(\mathrm{CVp})$ and offset $(\mathrm{CVo})$ variability. The participants were allocated to an experimental group $((\mathrm{n}=12$ and $\mathrm{n}=10)$ following a different strengthening program- or to the control group $(n=9)$. No differences were identified in the frontal and transverse plane. However, differences reached statistical significance in the sagittal plane in the control groups indicating an improved repeatability of the movement pattern. However, the reflective markers were placed only in T12 and S1 spinous processes rather failing to sufficiently describe the movement of the lower back. In addition, the frequency of the intervention protocol was clinically inadequate.

A particularly comprehensive clinical trial was conducted by Papadakis et al. [25] who investigated movement variability of 35 patients with lumbar stenosis in comparison to healthy individuals during a $40 \mathrm{~m}$ normal walking. A 3-axial accelerometer at $128 \mathrm{~Hz}$ was placed on the spinous process of L5. Only the vertical z-axis acceleration was used and differential entropy algorithm -which is an extension of Shannon's entropy to continuous variables- was implemented. The experimental group indicated higher values of entropy exhibiting higher 'movement irregularity' levels. These findings are attributed to a possible reformation of the gait pattern resulting in irregular and unpredictable movement behavior [25]. Furthermore, the Receiver Operating Characteristic (ROC) (graphical plot that illustrates the diagnostic ability of a binary classifier system as its discrimination threshold is varied) successfully identified $97.1 \%$ of the LBP group and $80.0 \%$ of the healthy participants reaching a $97.6 \%$ of distinguishing the two groups. In addition, the Oswestry Low Back Questionnaire seems to be well correlated with the differential entropy values. 
The aim of the present study was to investigate movement variability in LBP population in comparison to healthy subjects during gait. More specifically, the amount of chaos in the movement pattern of the lower limb, pelvis and lumbar spine of the LBP group was compared to the control group. It is expected that the LBP group will present with lower ApEn and/ or LyE values, underlying the less chaotic nature of their gait pattern. Gender differences in movement variability were also investigated.

\section{Materials and Methods}

\section{Participants}

Sixteen LBP patients and thirteen healthy control subjects (non- athletes) participated in the present study. This number of participants is quite sufficient for performing gait analysis17. The subjects were sufficiently matched in terms of somatometric features, gender $(10 / 16(62.5 \%)$ vs $8 / 13(61.5 \%)$ males in the LBP and healthy group respectively) and age ( $45.6 \pm 9.9$ vs $44.0 \pm 6.4$ years in the LBP and healthy group respectively). This specific age group is the most representative of the LBP population, in need for constant medical and physiotherapy treatment [4]. In addition, specific therapeutic guidelines have been proposed for this specific patient group [26]. Participants with localized lumbar pain persisting for longer than 6 months were included. Participants referring acute or radiating pain were excluded. After having been informed about the scope and methods of the present study the participants signed the Informed Consent Form. All participants were asked to complete the Oswestry Disability Index [27] and Roland-Morris Disability Questionnaire [28].

\section{Measurement procedures}

Participants walked barefoot on a motorized treadmill for 30 seconds (Pegasus ${ }^{\circledR}$ F-300, 3hp, $51 \mathrm{~cm} \chi 140 \mathrm{~cm}$ [29]) at 3 different walking conditions:

Condition A: Walking at a speed chosen by the subject with no inclination of the treadmill.

Condition B: Walking at a speed of $5 \mathrm{Km} / \mathrm{h}$ with no inclination of the treadmill.

Condition C: Walking at a speed of $5 \mathrm{Km} / \mathrm{h}$ with an inclination of $7^{\circ}$ of the treadmill $[30,31]$.

2 attempts were performed for each condition. Prior to the initiation of the recording participants had a $6 \mathrm{~min}$ familiarization period on the treadmill [32].

Measurements were taken from two positions: Pelvis and Lumbar simultaneously for three components (X, Y, and Z). In the lumbar position the $\mathrm{X}$ component signifies the lateral flexion, the $\mathrm{Y}$ component is flexion-extension and the $\mathrm{Z}$ component measures the axial rotation. In the pelvis the $\mathrm{X}$ component is the lateral tilt (elevation-depression), the Y component is the anterior-posterior tilt and the $\mathrm{Z}$ component is again the axial rotation.
Each measurement was performed for about 30 seconds and the recording was performed at a rate of 100 frames per second, yielding in each case a time series of around 3000 values. From each time series a time window of 2800 values were taken from 101 to 2900 frames. For each time window three parameters were evaluated: the linear parameter of Standard Deviation (SD) and the non-linear parameters of Approximate Entropy (ApEn) and Maximal Lyapunov Exponent (LyE). All calculations were performed with the R software package.

\section{Data acquisition}

The 6- infrared camera optoelectronic system Vicon (Vicon Motion Systems Ltd, UK) of ELEPAP (Gait \& Motion Analysis LabAthens) was used for the 3D kinematic data acquisition using the Vicon Nexus software. The specific system has been characterized as the 'gold standard' in gait analysis and has been used by numerous researchers in the last years [24,25]. The sampling rate was set at $100 \mathrm{~Hz}$ and the data were used unfiltered to avoid unnecessary normalization. Reflective markers were placed on specific anatomic landmarks according to the Plug-In-Gait model by the same examiner. More specifically, markers were placed on the skin surface of both anterior superior iliac spines, mid thighs, lateral femoral epicondyles, mid tibias, lateral malleoli, outsole of the foot, at the second metatarsal heads and heels. As far as the lumbar spine is concerned reflective markers were placed on the spinous processes of Lumbar 1 (L1), on the transverse process of L3 and on the upper end of the sacrum (S1- Crosbie et al. [30]). The lumbar spine was defined by the relative movement of L1 on S1 while L3 was used as a guide marker to identify lumbar rotations. The above modeling sufficiently describes lumbar movement minimizing the loss of data due to subcutaneous tissue displacement. However, separating the upper from lower lumbar spine (LS) is not feasible, although for the purposes of the present study this might not be a priority as we focus on the structure of lumbar movement and not its exact biomechanical behavior [33,34].

\section{Statistical analysis}

Reliable measurements throughout the total time window of 2800 frames were obtained for 23 subjects, 10 controls and 13 LBP patients. Each of the three measured parameters (SD, ApEn and LyE) yielded 18 calculated variables ( 3 conditions $\mathrm{X} 2$ positions $\mathrm{X}$ 3 components), where conditions, positions and components are explained above. Thus, there were 54 variables, each calculated twice. Preliminary analysis proved that the repeated measurements for each variable did not differ significantly, thus providing a further validation of the measurement and calculation methods. Consequently, the average values for each of the repeated values were taken.

The between groups and between gender differences of the calculated components were evaluated with the independent samples t-test. Statistical significance was set at 0.05 . For the 
most significant component ROC analysis for the calculation of its discrimination ability between the two groups was performed. This was followed by establishing the Youden index, i.e. the cutoff value that yields the best tradeoff between sensitivity and specificity for the discrimination of the two groups $(\mathrm{J}=\operatorname{maxc}\{\operatorname{Se}(\mathrm{c})+\operatorname{Sp}(\mathrm{c})-\})$.

\section{Results}

Table 1: Statistically significant differences in all 3 components (X, Y, Z) between LBP and controls in descending order of significance.

\begin{tabular}{|c|c|c|c|c|c|c|}
\hline Order & Condition & Position & Component & Parameter & t-value (df=21) & p-value \\
\hline 1 & A & Pelvis & X & LyE & 4.316 & 0.0003 \\
\hline 2 & A & Lumbar & X & SD & 3.618 & 0.002 \\
\hline 3 & A & Lumbar & X & LyE & 3.15 & 0.005 \\
\hline 4 & C & Lumbar & Y & ApEn & 3.023 & 0.006 \\
\hline 5 & B & Lumbar & Y & ApEn & 2.62 & 0.016 \\
\hline 6 & A & Pelvis & Y & LyE & 2.187 & 0.04 \\
\hline 7 & B & Lumbar & Z & ApEn & 2.18 & 0.041 \\
\hline
\end{tabular}

Seven variables from a total of 54 yielded statistically significant differences between the means of the controls and LBP subjects independent samples t-test. In four of them the significance level was less than 0.01 , where chance would allow such differences in not more than one variable. Another important observation is that the three most significant differences were observed for component $\mathrm{X}$ in condition A (Table 1).

In all seven components the mean values for the controls were significantly greater than for the LBP subjects (Figure 1).

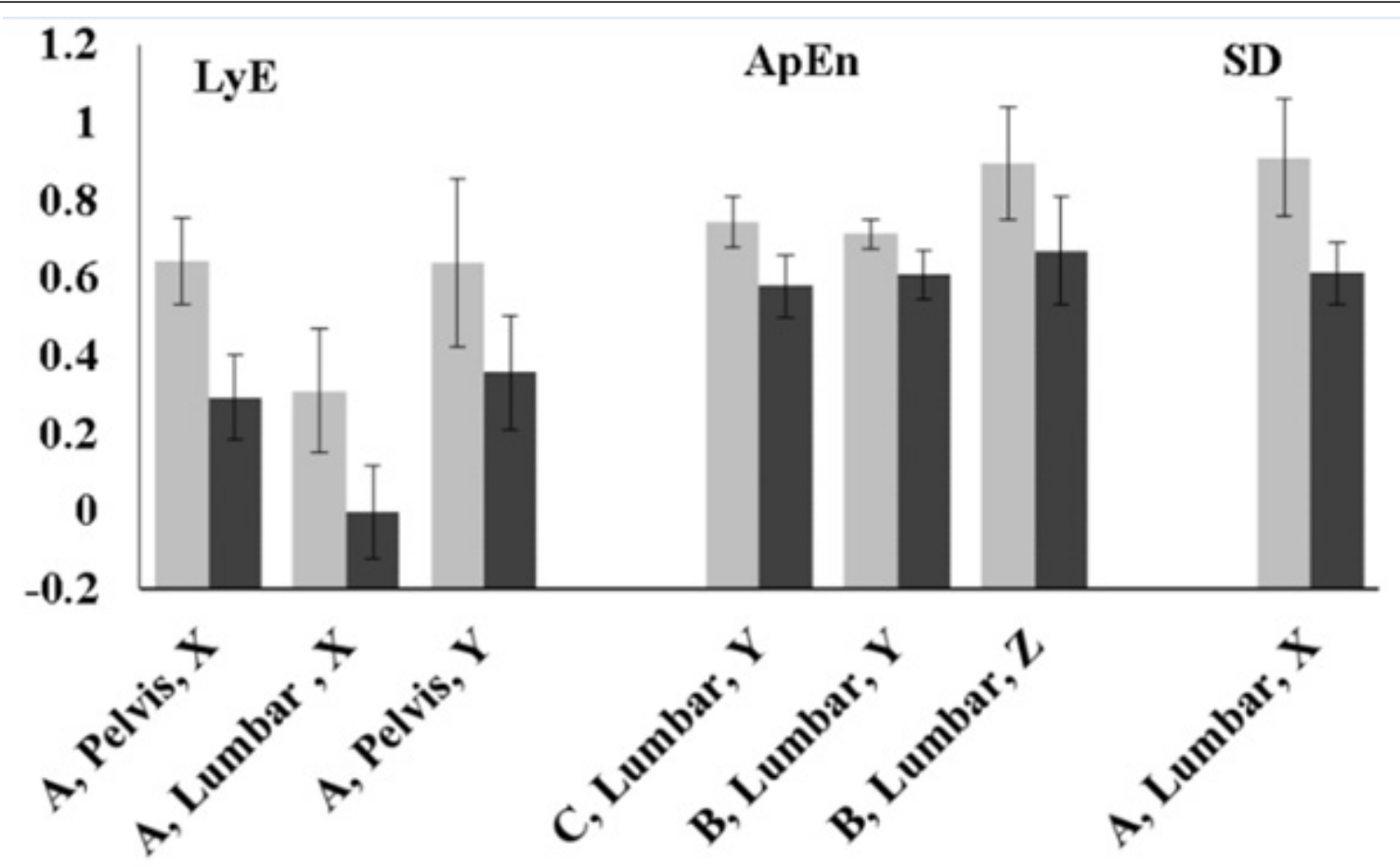

Control group a LBP group

Figure 1: Mean values of the two groups for the seven components. The axis labels (e.g. A, Pelvis, X) show the condition, position and component. The error bars are the standard errors of the means.

It is interesting that all the above components were also dependent on gender, with females having statistically significantly greater mean values than males. For example, for the Lye component, in condition A, position Pelvis, component $\mathrm{X}$ the mean value for the eleven female subjects was $0.60 \pm 0.24$, while for the sixteen male subjects the mean value was only $0.37 \pm 0.24(\mathrm{t}(\mathrm{df}=25)=2.4, \mathrm{p}=0.22)$. Nevertheless, the more important differences due to low back pain propagate to both genders in the same manner (Figure 2). 


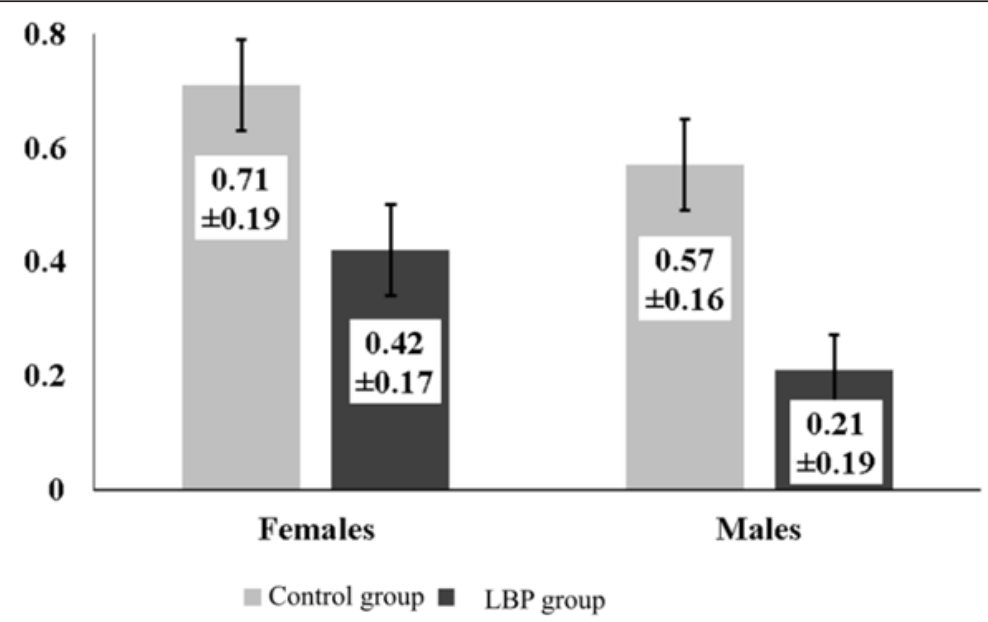

Figure 2: Mean values of the females and males of controls and experimental subjects for the Lye component, in condition A, position Pelvis, component X. The error bars are the standard errors of the means.

The Lye component, in condition A, position Pelvis, component controls from LBP subjects (Figure 3). The area under the curve $\mathrm{X}$ by itself has a very significant ability to discriminate healthy (AUC) was $0.900, \mathrm{p}<0.001$.

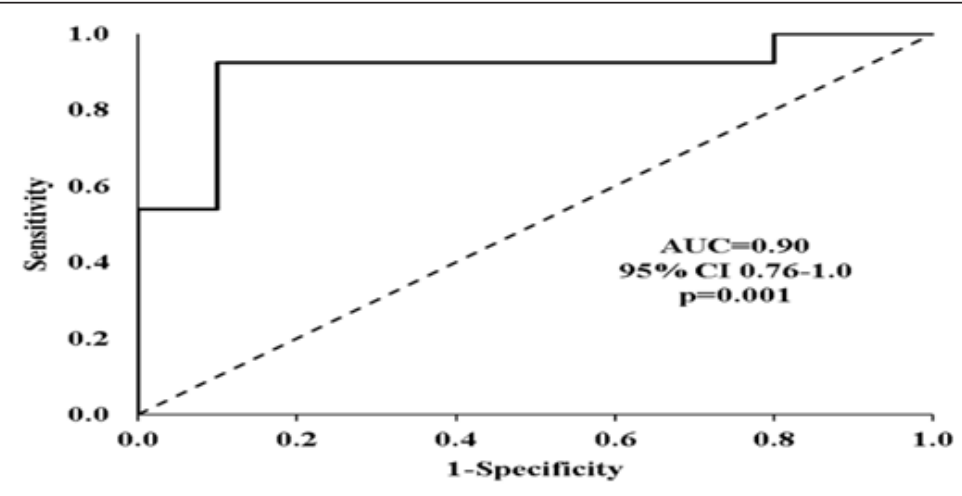

Figure 3: ROC curve for the discrimination ability of the Lye component, in condition A, position Pelvis, component $X$. Shown are the area under the curve (AUC) and its 95\% confidence intervals (CI) and its significance.

According to the Youden index the cutoff value that yielded discrimination ability between controls and LBP subjects was 0.52 the best tradeoff between sensitivity and specificity for the (Figure 4).

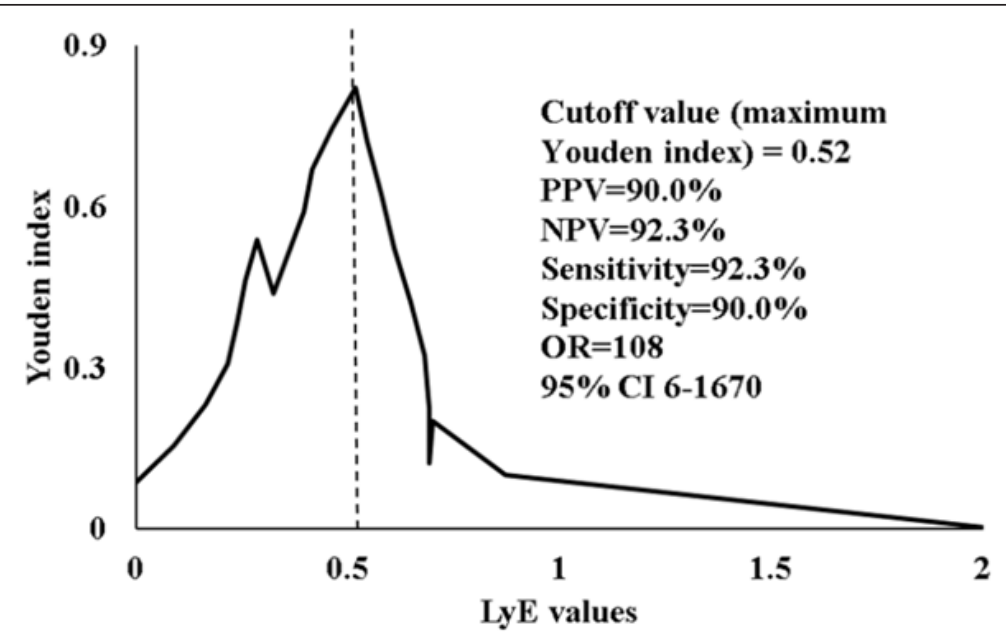

Figure 4: Youden index depending on the LyE values of the Lye component, in condition A, position Pelvis, component $\mathrm{X}$. 
Ten subjects with a Lye value greater than 0.52 were identified, nine of which were in the control group. This gives a negative predictive value (NPV) of $90 \%$. Similarly, there were thirteen subjects with a LyE equal to or less than 0.52 , twelve of which were LBP subjects, yielding a positive predictive value of $92.3 \%$. The sensitivity and specificity were also quite high $(92.3 \%$ and $90 \%$ respectively). This means that the calculation of just one LyE value in condition A, position Pelvis, component X can practically lead to an almost perfect differential diagnosis of the presence of LBP.

\section{Discussion}

The aim of the present study was to investigate movement variability in LBP population in comparison to healthy subjects during gait using linear (SD) and non-linear (Lye and ApEn) indices. Results showed that healthy participants presented with higher LyE, ApEn and SD values indicating higher variability levels and, therefore, a more chaotic kinematic behavior in comparison to LBP patients.

The outcomes of the present study align with the theory of Optimal Movement Variability since a more chaotic movement pattern for the healthy participants in comparison to the LBP sufferers is described. As far as the lumbar spine is concerned the control group presented with higher LyE and ApEn values indicating higher variability levels and, therefore, a richer movement repertoire with enhanced feedback. LyE values of the lumbar spine are in absolute accordance to the pelvis, both describing the movement pattern of the lumbo-pelvic complex. The differences are found in both the coronal and frontal plane. The ApEn values for the control decrease in conditions $B$ and $C$ where the neuromuscular activation and coordination demands are higher, and a more integrated proprioceptive feedback is essential. During gait a healthy individual is capable of dealing with more 'unstable' situations as they can apply a wider number of kinematic responses. On the contrary, LBP sufferers seem to adopt a stiffening strategy which disables them from adapting to random environmental disturbances render them susceptible to injury.

Similar to the findings of the present study Williams et al. [35] supported that participants with LBP avoid end of Range Of Motion (ROM) positions in an attempt to minimize the emerging pain by modifying their movement pattern as a result of inhibition or in order to achieve better active control. The absence of movement regularity- smoothness is the outcome of reduced proprioceptive income which derives from the underlying pathology [35]. Angular velocity and acceleration are significantly decreased in LBP population. However, ROM does not correlate with functional capability [36-38]. The above researchers also outlined that the quality of the lumbar movement pattern defines the incidence of LBP rather than the absolute ROM values or any other descriptive kinematics35. Like in the present study the use of non- linear mathematics seems to correlate well to the quality characteristics of movement rather than quantitative parameters.
Using a linear tool (Winter's Coefficient of Variation- CoV) that measures the repeatability of a movement pattern Vogt et al. [39] reported increased variability in LBP patients in the thorax and pelvis. The results of Vogt et al are in correspondence to the present one thus in $\alpha$ reverse correlation as it describes a more unstable and less effective movement pattern for the LBP group to all planes. In the present study the LS correlates to the pelvis while in the study of Vogt et al connects LS to the thorax. In addition, the researchers attributed the chronicity of the disease to neuromuscular dysfunction like agonist- antagonist coordination that result in amplified energy consumption [39]. According to Dingwell et al. [40] reduced or inadequate time, space or movement afferent feedback directly affects gait coordination resulting in an increase in the movement variability. Pain inhibition causes poor coordination in the entire lower limb- pelvis- lumbar spine complex, decreasing muscle efficacy and increasing fatigue levels [8]. Nevertheless, LBP patients counterbalance these deficits by recruiting compensatory movements during gait managing to reduce energy expenditure [41].

Based on the theory of Optimal Movement Variability $[7,16]$ described the ideal connection between predictability and complexity in gait as an inverted U- shape. The ideal movement variability is placed on top of the $U$ - shape suggesting that reducing variability does not axiomatically entail reduction in gait stability. Kaipust et al. [16] demonstrated that the incremental modification of the fractal properties during gait becomes less complex when a stable and periodic auditory stimulus is imposed. In our case, auditory stimulus is replaced by LBP condition. In comparison to our study LBP participants presented lower variability values possibly to avoid painful ROM or in order to effectively control locomotion [16].

According to numerous researchers each system is characterized by an inherent variability of deterministic nature which is defined by the underlying mechanisms of the system $[15,42,43]$. This variability states the formation and perception of the movement pattern transfusing complexity to the system and enabling it to counterbalance random external perturbations [43]. Pathologic perturbations as well as age are connected to lower variability levels resulting in complexity reduction and insufficient functional adaptability [43].

Chaos is definitely an essential component of healthy gait pattern. However, what is the ideal amount of variability that a kinematic system should have? Does higher variability entail a more stable kinetic behavior? The concept of Optimal Movement Variability seems to provide a sufficient theoretical frame for the above questions. However, defining the exact boundaries of the amount of variability for a given movement pattern yet remains to be done.

In various pathologies, like in LBP, the amount of variability is altered. The question yet remains whether variability increases 
or decreases. Researchers support either ways. Future studies are called to quantify the exact movement variability fluctuations each pathology induces.

From a clinical perspective, restoring the ideal amount of variability after its loss is the aim of every rehabilitation protocol. Proprioception training seems to be showing the way. Nevertheless, exercise programs are rather 'blind' since the clinician fails to evaluate the amount and quality of the training needed. Measuring movement variability in a clinical environment in a valid and reliable way must be a future priority for the research community.

\section{Conclusion}

In conclusion, healthy participants presented with higher LyE, ApEn and SD values indicating higher variability levels and, therefore, a more chaotic kinematic behavior in comparison to LBP patients. Lower variability values may be partly explained by the attempt of LBP patients to avoid painful end of range of motion positions. In this perspective non- linear indices seem to relate to qualitive characteristics of movement that need to be taken into consideration during rehabilitation.

\section{Acknowledgement}

We would like to express our gratitude to Onassis Foundation for its kind and valuable ethical and economic support to the accomplishment of the present study.

\section{References}

1. Carragee E, Babak B, Todd A, Van den Haak E (2004) Prospective controlled study of the development of lower back pain in previously asymptomatic subjects undergoing experimental discography. Spine 29(10): 1112-1117.

2. Caballero B (2007) The global epidemic of obesity: An overview. Epidemiologic Reviews 29: 1-5.

3. Bell JA, Burnett A (2009) Exercise for the primary, secondary and tertiary prevention of low back pain in the workplace: A systematic review. J Occup Rehabil 19: 8-24.

4. Cassidy JD, Cote P, Carroll L, Kristman V (2005) Incidence and course of low back pain episodes in the general population. Spine 30(24): $2817-$ 2823.

5. Brumagne S, Cordo P, Verschueren S (2004) Proprioceptive weighting changes in persons with low back pain and elderly persons during upright standing. Neuroscience Letters 366: 63-66.

6. Oddsson LIE, DeLuca CJ (2003) Activation imbalances in lumbar spine muscles in the presence of chronic low back pain. Journal of Applied Physiology 94: 1410-1420.

7. Stergiou N, Harbourne RT, Cavanaugh JT (2006) Optimal movement variability: A new theoretical perspective for neurologic physical therapy. Journal of Neurologic Physical Therapy 30(3): 120-129.

8. Schmidt RA, Lee TD (2005) Motor control and learning: A behavioral emphasis. ( $4^{\text {th }}$ edn), Human Kinetics Publishers, Champaign, USA.

9. Hubler A (1989) Adaptive control of chaotic systems. Swiss Physical Society. Helvetica Physica Acta 62: 339-342.

10. Stergiou N, Moraiti CO, Giakas G, Ristanis S, Georgoulis AD (2004) The effect of the walking speed on the stability of the anterior cruciate ligament deficient knee. Clin Biomech 19(9): 957-963.
11. Sosnoff JJ, Newell KM (2006a) Aging, visual intermittency, and variability in isometric force output. J Gerontol B Psychol Sci 61: 117-124.

12. Hausdorff JM (2009) Gait dynamics in parkinson's disease: common and distinct behavior among stride length, gait variability, and fractal-like scaling. Chaos 19(2): 026113.

13. Bruijn SM, Millard M, van Gestel L, Meyns P, Jonkers I, Desloovere K (2013) Gait stability in children with cerebral palsy. Res Dev Disabil 34: 1689-1699.

14. Mehdizadeh S (2018) The largest Lyapunov exponent of gait in young and elderly individuals: A systematic review. Gait \& Posture 60: 241-250.

15. Dingwell JB, Cusumano JP (2000) Nonlinear time series analysis of normal and pathological human walking. Chaos 10(4): 848-863.

16. Kaipust JP, McGrath D, Mukherjee M, Stergiou N (2013) Gait variablility is altered in older adults when listening to auditory stimuli with differing temporal structures. Ann Biomed Eng 41(8): 1595-1603.

17. Moraiti CO, Stergiou N, Vasiliadis HS, Motsis E, Georgoulis A (2010) Anterior cruciate ligament reconstruction results in alterations in gait variability. Gait \& Posture 32(2): 169-175.

18. Myers SA, Johanning JM, Stergiou N, Celis RI, Robinson L, et al. (2009) Gait 605 variability is altered in patients with peripheral arterial disease. J Vasc Surg 49(4): 924-931.

19.Zampeli F, Moraiti C, Xergia S, Tsiaras V, Stergiou N (2010) Stride-tostride variability is altered during backward walking in anterior cruciate ligament deficient patients. Clin Biomech 25(10): 1037-1041.

20. Courtney C, Rine RM, Kroll P (2005) Central somatosensory changes and altered muscle synergies in subjects with anterior cruciate ligament deficiency. Gait Posture 22: 69-74.

21. Thorstensson A (1986) How is the normal locomotor program modified to produce backward walking? Exp Brain Res 61: 664-668.

22. Tsepis E, Giakas G, Vagenas G, Georgoulis A (2004) Frequency content asymmetry of the isokinetic curve between ACL deficient and healthy knee. Clin Biomech 37: 857-864.

23. Moraiti CO, Stergiou N, Ristanis S, Georgoulis AD (2007) ACL deficiency affects stride to stride variability as measured using nonlinear methodology. Knee Surg Sports Traumatol Arthrosc 15(12): 1406-1413.

24. Steele J, Bruce-Low S, Smith D, Jessop D, Osborne N (2016) A randomized controlled trial of the effects of isolated lumbar extension exercise on lumbar kinematic pattern variability during gait in chronic low back pain. PM\&R 8(2): 105-114.

25. Papadakis NC, Christakis DG, Tzagarakis GN, Chlouverakis GI, Kampanis NA, et al. (2009) Gait variability measurements in lumbar spinal stenosis patients: part B. Preoperative versus postoperative gait variability. Physiol Meas 30(11): 1187-1195.

26. Chou R, Qaseem A, Vincenza S, Casey D, Cross T, et al. (2007) Diagnosis and treatment of low back pain: A joint clinical practice guideline from the american college of physicians and the american pain society. Annals of Internal Medicine 147(7): 478-491.

27. Fairbank JC, Couper J, Davies JB, O'Brien JP (1980) The oswestry low back pain disability questionnaire. Physiotherapy 66: 271-273.

28. Roland M, Morris R (1983) A study of the natural history of back pain: Part I. Development of a reliable and sensitive measure of disability in low-back pain. Spine 8(2): 141-144.

29. Lugade V, Lin V, Chou LS (2011) Center of mass and base of support interaction during gait. Gait \& Posture 33: 406-411.

30. Crosbie J, de Faria Negrao Filho R, Nascimento DP, Ferreira P (2013) Coordination of spinal motion in the transverse and frontal planes during walking in people with and without recurrent low back pain. Spine 38(5): 286-292. 
31. Ehlen KA, Reiser II RF, Browning RC (2011) Energetics and biomechanics of inclined treadmill walking in obese adults. Medicine \& Science in Sports \& Exercise 43(7): 1251-1259.

32. Zeni JA, Higginson JS (2010) Gait parameters and stride-to-stride variability during familiarization to walking on a split-belt treadmill. Clinical Biomechanics (Bristol, Avon) 25(4): 383-386.

33. Dankaerts W, O’Sullivan P, Burnett A, Straker L (2006) Differences in sitting postures are associated with non-specific chronic low back pain disorders when patients are subclassified. Spine 31(6): 698-704.

34. Mitchell T, O’Sullivan PB, Burnett AF, Straker L, Rudd C (2008) Low back pain characteristics from undergraduate student to working nurse in Australia: A cross-sectional survey. International Journal of Nursing Studies 45(11): 1636-1644.

35. Williams JA, Haq I, Lee RY (2013) A novel approach to the clinical evaluation of differential kinematics of the lumbar spine. Manual Therapy 18(2): 130-135.

36. Parks KA, Crichton KS, Goldford RJ, McGill SM (2003) A comparison of lumbar range of motion and functional ability scores in patients with low back pain: assessment for range of motion validity. Spine 28(4): 380-384.

37. Shum GLK, Crosbie J, Lee RYW (2005a) Effect of low back pain on the kinematics and joint coordination of the lumbar spine and hip during sit-to-stand and stand-to-sit. Spine 30(17): 1998-2004.
38. Shum GLK, Crosbie J, Lee RYW (2005b) Symptomatic and asymptomatic movement coordination of the lumbar spine and hip during an everyday activity. Spine 30(23): E697-702.

39. Vogt L, Pfeifer K, Portscher M, Banzer W (2001) Influences of nonspecific low back pain on three-dimensional lumbar spine kinematics in locomotion. Spine 26: 1910-1919.

40. Dingwell J, Robb, R, Troy K, Grabiner M (2008) Effects of an attention demanding task on dynamic stability during treadmill walking. Journal of Neuroeng Rehabil 5(1): 12.

41. Waters RL, Mulroy S (1999) The energy expenditure of normal and pathologic gait. Gait Posture 9(3): 207-231.

42. Hausdorff JM, Peng CK, Ladin Z, Wei JY, Goldberger AL (1995) Is walking a random walk? Evidence for long-range correlations in the stride interval of human gait. J Appl Physiol 78(1): 349-358.

43. Goldberger AL, Amaral LA, Hausdorff JM, Ivanov PC, Peng CK, et al. (2002) Fractal dynamics in physiology: alterations with disease and aging. Proceedings of the National Academy of Sciences 99(Suppl 1): 2466-2472. 